\title{
Performance Analysis for Cooperative Communication System with QC-LDPC Codes Constructed with Integer Sequences
}

\author{
Yan Zhang, ${ }^{1,2}$ Feng-fan Yang, ${ }^{1}$ and Weijun Song ${ }^{2}$ \\ ${ }^{1}$ College of Electronic and Information Engineering, Nanjing University of Aeronautics and Astronautics, Nanjing, \\ Jiangsu 210016, China \\ ${ }^{2}$ Nanjing College of Information Technology, Nanjing, Jiangsu 210046, China \\ Correspondence should be addressed to Yan Zhang; zhangyanncit@163.com
}

Received 19 October 2014; Revised 10 December 2014; Accepted 25 December 2014

Academic Editor: Muhammad Naveed Iqbal

Copyright (c) 2015 Yan Zhang et al. This is an open access article distributed under the Creative Commons Attribution License, which permits unrestricted use, distribution, and reproduction in any medium, provided the original work is properly cited.

\begin{abstract}
This paper presents four different integer sequences to construct quasi-cyclic low-density parity-check (QC-LDPC) codes with mathematical theory. The paper introduces the procedure of the coding principle and coding. Four different integer sequences constructing QC-LDPC code are compared with LDPC codes by using PEG algorithm, array codes, and the Mackey codes, respectively. Then, the integer sequence QC-LDPC codes are used in coded cooperative communication. Simulation results show that the integer sequence constructed QC-LDPC codes are effective, and overall performance is better than that of other types of LDPC codes in the coded cooperative communication. The performance of Dayan integer sequence constructed QC-LDPC is the most excellent performance.
\end{abstract}

\section{Introduction}

In 1979, Cover and El Gamal reached substantial progress in cooperative communication technology $[1,2]$. Amplify-andforward (AF) [3], decode-and-forward (DF) [4], and coded cooperation (CC) [5] are three commonly user cooperation modes. In coded cooperation, the relay user does not send signals of cooperative users repeatedly. With features of coding, this method sends different parts of codes, respectively. Due to the fact that different parts in codes have innate correlated characteristics, if the base station receives different parts of codes by mutually opposed channels, that is, if the information of each code is sent by two channels, the transmission diversity is realized and the coding gain is acquired. As the application of channel, coded cooperation mode is superior to the previous methods.

According to the characteristics of low-density paritycheck (LDPC) codes coded cooperative system, many scholars had achieved fruitful research on designing LDPC codeword construction in the coded cooperative system [6-8]. In single-source single relay scenario, the literature [9] proposed that using computer search structure could optimize LDPC code sets. In the multisource coded cooperative system, the literature [10] introduced that LDPC codes are optimized by distribution function without taking into account the complexity of encoding and decoding, memory, and other resource consumption. This optimized distribution function is long enough to clearly show the advantages of its performance. As the length of LDPC is large, the complexity of coding and decoding is too high and the memory consumption is larger in hardware implementation.

Quasi-cyclic low-density parity-check (QC-LDPC) codes coded cooperative system is an important subclass of LDPC. Compared with randomly constructed LDPC, QC-LDPC can achieve a simple shift register and linear coding complexity, but the parameters of the code are not flexible enough, and they cannot meet the needs of practical application.

In point-to-point (noncooperative) system, the literature [11] proposed various design methods of QC-LDPC; for example, the literature [12] designed the QC-LDPC with the ring length of at least 8 through the greatest common divisor inequality when the subblock length is greater than a lower bound. The literature [13] designed the QC-LDPC in coded cooperative system by finite field (Galois Field, GF) of the multiplicative inverses, but the check matrix of QC-LDPC is limited to the lower triangular structure. 


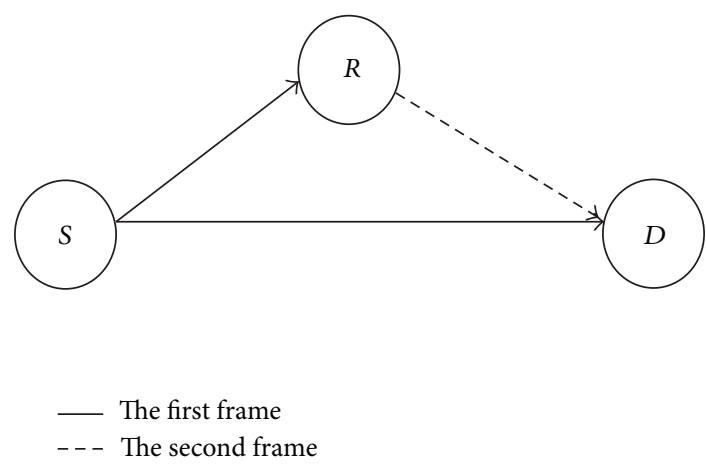

FIgURE 1: Coded cooperative communication model.

For this problem, this paper introduces the QC-LDPC constructed by four different integer sequences, compared with traditional QC-LDPC; the class QC-LDPC can save more space used with linear encoding complexity and quasicyclic structure. Through simulating, the results show that the QC-LDPC constructed by integer sequences is without the girth-4 length and is excellent in performance.

The rest of the paper is organized as follows. Section 2 introduces fundamentals of general coded relay cooperation. Section 3 presents QC-LDPC constructed by four different integer sequences. The simulation results are shown in Section 4. Finally, the conclusions are reached in Section 5.

\section{Fundamentals of General Coded Relay Cooperation}

In this paper, 3-nodes relay channel model is a common model to describe the cooperative communication system. It is shown in Figure 1. It is comprised of three fundamental communication units such as the source $S$, the relay $R$, and the destination $D$. All these terminals are assumed to have a single antenna and they communicate with each other in half-duplex mode.

The transmission of information data is divided into two frames. In the first frame, the source node $(S)$ encodes information using the $\operatorname{code} c_{1}$ and sends its information to the relay node $(R)$ and the destination node $(D)$ simultaneously. During the first frame the received signal $y_{S R}$ over a Rayleigh fading channel at the relay is given as

$$
y_{S R}=h_{S R} x_{A}+n_{S R}
$$

where $n_{S R}$ is complex additive Gaussian noise with each component as a zero-mean complex Gaussian variable with independent real and imaginary parts of equal variance $N_{0} / 2$. $h_{S R}$ is also complex Gaussian variables with zero-mean 1/2variance for the real and imaginary parts of each component. Similarly, the received signal $y_{S D}$ over a Raleigh fading channel at the destination during the first frame is described as

$$
y_{S D}=h_{S D} x_{A}+n_{S D},
$$

where $n_{S D}$ and $h_{S D}$ are corresponding to Gaussian noise and fading, respectively.
In the second frame, if the relay node can decode the information successfully, then using BPSK modulation the relay sends the coded information $c_{2}$ to the destination. Otherwise, the relay node does not work. $h_{i, j}$ and $n_{i, j}$ are were taken representatively as channel gain and additive noise of each channel. Each channel is mutually independent. The received signal $y_{R D}$ over a Rayleigh fading channel at the destination during the second frame is given as

$$
y_{R D}=h_{R D} x_{R}+n_{R D},
$$

where $n_{R D}$ and $h_{R D}$ are associated with Gaussian noise and fading, respectively. $x_{R}$ represents the information sent by relay node. Finally, the overall received signal $y$ at the destination is given as

$$
y=\left|y_{S D}\right| y_{R D} \mid,
$$

where $\left|y_{S D}\right| y_{R D} \mid$ is the series concatenation of $y_{S D}$ and $y_{R D}$ received in the first frame and second frame, respectively. The signal $y$ is jointly decoded to recover the transmitted information at the source.

In the information transmitting procedure, two different LDPC codes $C_{1}\left(K_{1}, N_{1}\right)$ and $C_{2}\left(K_{2}, N_{2}\right)$ were used to represent the source node $S$ and relay node $R . K_{i}$ and $N_{i}$, respectively, represent the dimension and length of codes. The length of coded message sequence of source node $S$ is $K_{1}$ and the check matrix is $H_{1} . C_{1}$ and $C_{2}$ can be designed for simple systematic codes. The code of $C_{1}$ is $\left(I, P_{1}\right)$, where $P_{1}$ is the parity bits with the length of $N_{1}-K_{1}$. If relay node succeeds to code after receiving information from $C_{1}$, relay node will generate check matrix $\mathrm{H}_{2}$ by adding new parity bits $P_{2}$ with the length of $N_{2}-K_{2}$. The destination node $D$ would receive information from source node $S$ and relay node $R$. After the combination, the code $\left(I, P_{1}, P_{2}\right)$ is obtained. The joint coding is conducted by the check matrix $H$ comprised of $H_{1}$ and $H_{2}$ to acquire source information.

The entire code of $C_{1}$ and parity bit of $C_{2}$, respectively, reach the destination node through $S-D$ channel and $R-D$ channel, which can be obtained by the check relationship of codes. Consider

$$
H_{1} C_{1}=0, \quad H_{2} C_{2}=0 .
$$

As can be seen from (5), the entire code of coded cooperative communication system can meet the following check relationship:

$$
H C=0 .
$$

\section{QC-LDPC Constructed by Four Different Integer Sequences}

The check matrix $H$ of regular $(J, L)$ QC-LDPC codes is expressed by $J \times L$ array of $p \times p$ cyclic permutation matrix. Its length is $n=L P$, which can be defined as

$$
H=\left[\begin{array}{cccc}
I\left(p_{0,0}\right) & I\left(p_{0,1}\right) & \cdots & I\left(p_{0, L-1}\right) \\
I\left(p_{1,0}\right) & I\left(p_{1,1}\right) & \cdots & I\left(p_{1, L-1}\right) \\
\vdots & \vdots & \vdots & \vdots \\
I\left(p_{J-1,0}\right) & I\left(p_{J-1,1}\right) & \ldots & I\left(p_{J-1, L-1}\right)
\end{array}\right],
$$


where $0 \leq j \leq J-1 ; 0 \leq l \leq L-1, I\left(p_{j, l}\right)$ is the $p \times p$ cyclic permutation matrix, and each row and line of the matrix contain only one 1 . The last line of the matrix is ring shifted right by one element so as to acquire the line one of the matrix; each column of the matrix is ring shifted downward by one element to obtain the next column of the matrix; the last column of the matrix is ring shifted downward by one element to obtain the first column of the matrix. For example, matrix $P$ is the $5 \times 5$ cyclic permutation matrix:

$$
P=\left[\begin{array}{lllll}
1 & 0 & 0 & 0 & 0 \\
0 & 1 & 0 & 0 & 0 \\
0 & 0 & 1 & 0 & 0 \\
0 & 0 & 0 & 1 & 0 \\
0 & 0 & 0 & 0 & 1
\end{array}\right] .
$$

$p_{i, j}$ represents the shift value of this cyclic permutation matrix; that is, 1 of $r$ th of this matrix was in the column $\left(r+p_{j, l}\right) \bmod p, 0 \leq r \leq p-1$, while the other columns of this row are $0 . I(0)$ represents the unit matrix with the size of $p \times p$, and $I\left(p_{j, l}\right)$ shows the matrix after the ring shift right of $I(0)$ by $p_{i, j}$. Assume the shift matrix $B$ of check matrix $H$ formed by $I\left(p_{i, j}\right)$ with the right shifting number $p_{i, j}$ as follows:

$$
B=\left[\begin{array}{cccc}
p_{0,0} & p_{0,1} & \cdots & p_{0, L-1} \\
p_{1,0} & p_{1,1} & \cdots & p_{1, L-1} \\
\vdots & \vdots & \vdots & \vdots \\
p_{J-1,0} & p_{J-1,1} & \cdots & p_{J-1, L-1}
\end{array}\right] .
$$

The above QC-LDPC codes check matrix contains a cycle, which could be expressed by the sequence of $p \times p$ cyclic permutation matrix; the cycle of QC-LDPC codes with the length of $2 i$ can be expressed as $\left(j_{0}, l_{0}\right),\left(j_{1}, l_{1}\right), \ldots$, $\left(j_{k}, l_{k}\right), \ldots,\left(j_{i-1}, l_{i-1}\right),\left(j_{0}, l_{0}\right)$, where $\left(j_{i}, l_{i}\right)$ is $I\left(p_{j, k}\right)$ of $H$ matrix at row $j_{k}$ and column $l_{k}$. To accurately express a cycle, $j_{k} \neq j_{k+1}$ and $l_{k} \neq l_{k+1}$ should be met. The literature [14] proposed that the necessary and sufficient condition for a code without girth 4 is

$$
\sum_{k=0}^{i-1}\left(p_{j_{k}, l_{k}}-p_{j_{k+1}, l_{k}}\right) \neq 0 \bmod p
$$

where $j_{i}=j_{0} ; j_{k} \neq j_{k+1} ; l_{k} \neq l_{k+1}$.

\subsection{QC-LDPC Codes Constructed by Fibonacci Array}

Definition 1 (see [15]). For an array $f(n), n$ is a nonnegative integer. If the array meets

$$
\begin{gathered}
f(n)=f(n-1)+f(n-2) \quad(n \geq 2), \\
f(0)=1, \quad f(1)=1 .
\end{gathered}
$$

Then, this array is known as Fibonacci array, and its element is Fibonacci value [16]. For example, a typical Fibonacci array is as follows: $1,1,2,3,5,8,13,21,34,55, \ldots$.

According to the check matrix in (7), the shift value of cyclic permutation matrix at row $j$ column $l$ is

$$
p_{j, l}=f(j+l+2)+j .
$$

Literature [17] verified that girth 4 of $H$ matrix designed by Fibonacci array can be removed. According to the above rules, it can be assumed that in the design of QC-LDPC of Fibonacci array of $H(10,1,2)$, each item of shift matrix was

$$
\begin{aligned}
& p_{0,0}=f(0+0+2)+0=f(2)=2, \\
& p_{0,1}=f(0+1+2)+0=f(3)=3 .
\end{aligned}
$$

Shift matrix $B$ can be written as follows: $B=\left[\begin{array}{ll}2 & 3\end{array}\right]$.

$p$ can flexibly select any value greater than the maximal Fibonacci value and $p$ is identified as shift. The maximal value in the matrix is +2 and $p=5$, and according to the definition of cyclic permutation, one has

$$
\begin{aligned}
H(10,1,2) & =\left[I\left(p_{0,0}\right)\right. \\
& \left.I\left(p_{0,1}\right)\right] \\
& =\left[\begin{array}{llllllllll}
0 & 0 & 1 & 0 & 0 & 0 & 0 & 0 & 1 & 0 \\
0 & 0 & 0 & 1 & 0 & 0 & 0 & 0 & 0 & 1 \\
0 & 0 & 0 & 0 & 1 & 1 & 0 & 0 & 0 & 0 \\
1 & 0 & 0 & 0 & 0 & 0 & 1 & 0 & 0 & 0 \\
0 & 1 & 0 & 0 & 0 & 0 & 0 & 1 & 0 & 0
\end{array}\right] .
\end{aligned}
$$

\subsection{The Design of QC-LDPC Codes with}

\section{Dayan Integer Sequence}

Definition 2 (see [18]). For an array $f(n)$, when $n$ is a nonnegative integer and an odd number, one has

$$
f(n)=\frac{(n \times n-1)}{2},
$$

and when $n$ is an even number, one has

$$
f(n)=\frac{(n \times n)}{2} .
$$

The sequence that satisfied the recurrence relation is known as Dayan integer sequence, and its element is the item of Dayan integer sequence. A typical Dayan integer sequence is as follows: $0,2,4,8,12,18,24,32,40,50, \ldots$.

In a Dayan integer sequence, the new sequence formed by Dayan integer sequence items of numbers with the same item number difference shows monotone increasing. Besides, QCLDPC constructed by Dayan integer sequence can remove girth 4 , and the verification process is shown in Literature [19]. In accordance with check matrix in (7), the shift equation of cyclic permutation matrix at row $j$ and line $l$ is

$$
p_{j, l}=f(j+l+l)+j .
$$

For example, to construct a check matrix $H(30,1,2)$, each item of corresponding shift matrix $B(1,2)$ is as follows:

$$
\begin{aligned}
p_{0,0} & =f(1+1+1)+1=f(3)+1 \\
& =\frac{(3 \times 3-1)}{2}+1=4+1=5, \\
p_{0,1} & =f(1+2+2)+1=f(5)+1 \\
& =\frac{(5 \times 5-1)}{2}+1=12+1=13 .
\end{aligned}
$$

The form to the shift matrix is $B(1,2)=\left[\begin{array}{ll}5 & 13\end{array}\right]$. 


\subsection{QC-LDPC Constructed by Differential Sequence}

Definition 3 (see [20]). Let $h_{0}, h_{1}, h_{2}, \ldots, h_{n}, \ldots$

As a digital sequence, thus, we define a new sequence $\Delta h_{0}, \Delta h_{1}, \Delta h_{2}, \ldots, \Delta h_{n}, \ldots$, known as first-order difference sequence, where

$$
\Delta h_{n}=h_{n+1}-h_{n}, \quad(n \geq 0) .
$$

In (20), each item of the difference sequence is the difference value of adjacent items in sequence (19). After the construction of difference sequence (20), the second-order difference sequence can be easily obtained:

$$
\Delta^{2} h_{0}, \Delta^{2} h_{1}, \Delta^{2} h_{2}, \ldots, \Delta^{2} h_{n}, \ldots
$$

Hence, one has

$$
\begin{aligned}
\Delta^{2} h_{n} & =\Delta\left(\Delta h_{n}\right)=\Delta h_{n+1}-\Delta h_{n} \\
& =\left(h_{n+2}-h_{n+1}\right)-\left(h_{n+1}-h_{n}\right) \\
& =h_{n+2}-2 h_{n+1}+h_{n} \quad(n \geq 0),
\end{aligned}
$$

where $\Delta^{m} h_{n}=\Delta\left(\Delta^{m-1} h_{n}\right)$.

Hence, $m$-order differential sequence is the first-order differential sequence of $m-1$-order differential sequence. We define the zero-order differential sequence as the digital sequence, as $\Delta^{0} h_{n}=h_{n},(n \geq 0)$. Assume $(3, k)$ QC-LDPC codes, known as DS-LDPC codes. The check matrix of this kind of codes consisted of zero-, first-, and second-order differential sequences generated by the quadratic polynomial. The design process of check matrix is shown in the literature [21].

\subsection{QC-LDPC Constructed by Hoey Sequence}

Definition 4 (see [22]). In a Hoey sequence, $h_{i}$ shows monotone increasing and meets the minimal nonnegative integer sequence with the sum of the two being different.

For $i=0,1, \ldots, 49$, the top 50 elements of $h_{i}$ are

$0,1,3,7,12,20,30,44,65,80,96,122,147,181,203,251$,

$289,360,400,474,564,661,774,821,915,969,1015,1158$,

$1311,1394,1522,1571,1820,2028,2253,2509,2779,2924$,

3154, 3353, 3590, 3796, 3997, 4296, 4432, 4778, 4850.

Let the form of shift matrix $B$ of QC-LDPC codes be as follows:

$$
B=\left[\begin{array}{cccc}
h_{0} & h_{1} & \cdots & h_{L-1} \\
0 & 0 & \cdots & 0
\end{array}\right]
$$

Elements in the first row of shift matrix $B$ are derived from top $L$ elements in Hoey sequence and each element in the second row is 0 . Shift matrix $B$ is a $2 \times L$ matrix, and

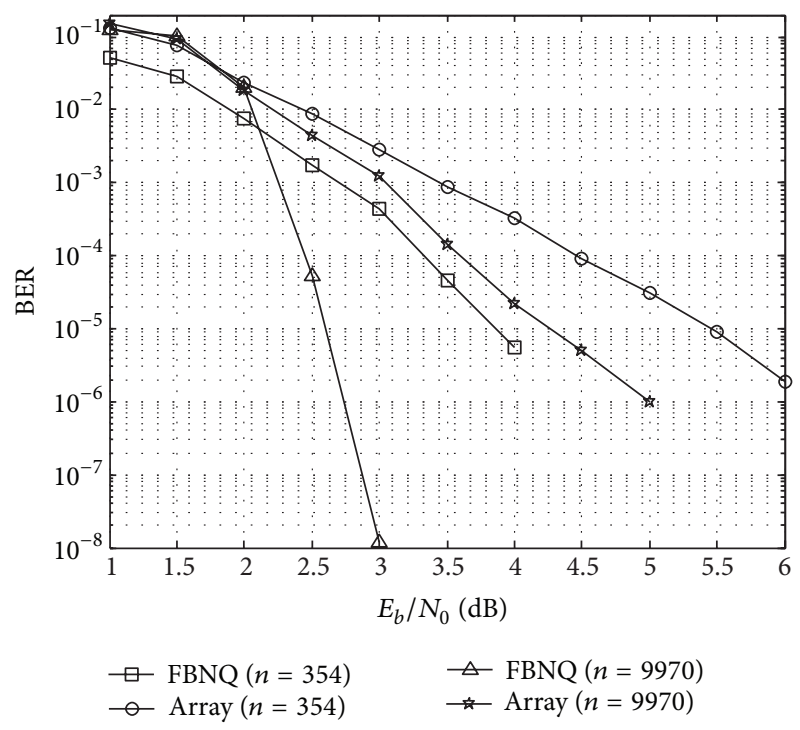

FIGURE 2: The curves of Fibonacci-constructed QC-LDPC and QCLDPC codes BER (bit error rate) constructed by array sequence.

the corresponding check matrix $H$ is a $2 \times L$ array formed by $p \times p$ cyclic permutation matrix, where $p>\max _{0 \leq i \leq L-1}\left\{h_{i}\right\}$ and the null space of $H$ presents a $(2, L)$ regular QC-LDPC code with the length of $p L$ and the code rate of at least $(p L-$ $2 p+1) / p L$. The check matrix constructed by Hoey sequence does not include girth 4 , which was verified by the literature [23]. With the example of the minimum matrix, due to the fixed pattern of base matrix of Hoey sequence, we obtain (23).

Thus, the minimal matrix is $2 * 4$ matrix. Consider

$$
B=\left[\begin{array}{llll}
0 & 1 & 3 & 7 \\
0 & 0 & 0 & 0
\end{array}\right] \text {. }
$$

\section{Simulation Results}

In this section, we select the $(3,6)$ Fibonacci array with code rate $R=1 / 2$ and a length of 354 to construct QC-LDPC codes $(p=59)$, as well as the $(5,10)$ Fibonacci with the length of 9970 to construct QC-LDPC codes $(p=997)$, which were compared with array LDPC codes [24] with the same length in the Gaussian white noise channel. The maximum number of iterations is 50 .

As can be seen from Figure 2, performance of QC-LDPC codes constructed by Fibonacci with the length of 354 is better than that of array LDPC codes. When BER $=4 \times 10^{-6}$, the performance of Fibonacci-constructed QC-LDPC codes is improved by about $1.6 \mathrm{~dB}$ compared to the performance of array LDPC codes. The QC-LDPC codes constructed by Fibonacci with the length of 9970 are superior to array LDPC codes with the same parameters.

For the simulation of QC-LDPC code constructed by differential sequence, a quadratic polynomial $h_{n}=2 n^{2}+3 n+1$ is found by the search algorithm. As $\max _{0 \leq i \leq 5, j \in\{0,1,2\}}\left\{\Delta^{j} h_{i}\right\}=$ $66, p=67$, and a $67 \times 67$ cyclic permutation matrix formed $3 \times 6$ array $H$. $H$ matrix is a $201 \times 402$ matrix. The column weight is 3 and the row weight is 6 . In order to facilitate 


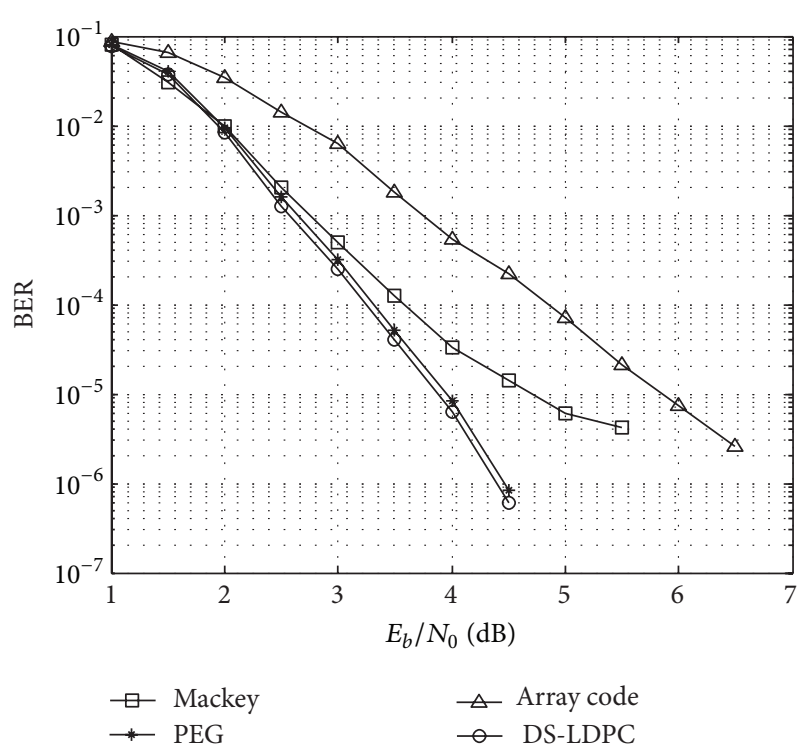

FIGURE 3: The BER curve comparison between QC-LDPC constructed by differential sequences, Mackay, PEG algorithm, and array codes.

the comparison, the PEG algorithm [25] is used to construct the pseudorandom $(402,201)$ PEG codes. Besides, the random $(402,201)$ MacKay codes (see [26]) and a $(402,203)$ array codes are constructed (see [27]), and the performance is compared as in Figure 3.

As can be seen from Figure 3, at BER $=10^{-5}$, Mackay codes began to show platform, whereas DS-LDPC codes did not show platform at BER $=10^{-7}$. At BER $=10^{-5}$, DS-LDPC codes comparing with Mackay codes and array codes, there are about $0.7 \mathrm{~dB}$ and $2.0 \mathrm{~dB}$ coding gain. The performance of differential sequence is better than that of MacKay, PEG algorithm, and array codes in the condition of the same parameters.

With the performance simulation of QC-LDPC codes constructed by Hoey sequence, let $L=12$ and $p=123$, and a $2 \times 12$ shift matrix can be constructed to make a $2 \times$ 12 array $H$ constructed by a $123 \times 123$ cyclic permutation matrix. $H$ is a $246 \times 1476$ matrix, with the column weight and row weight of 2 and 12, respectively. Its null space provides $(1476,1231)$ binary QC-LDPC codes with the code rate of 0.83 . The performance comparison and analysis of $(1476,1231)$ pseudorandom PEG codes and the $(1476,1231)$ array codes are as in Figure 4.

As can be seen from Figure 4, at BER $=10^{-5}$, Hoey code compared with array code improves about $1.1 \mathrm{~dB}$ coding gain. The performance of QC-LDPC codes constructed by Hoey sequence is better than that of the QC-LDPC codes constructed by PEG algorithm and that of QC-LDPC codes constructed by array code in the condition of the same parameters.

QC-LDPC constructed by four different integer sequences, respectively, is used in the code cooperative communication system. The system model is shown in Figure 1. Assume

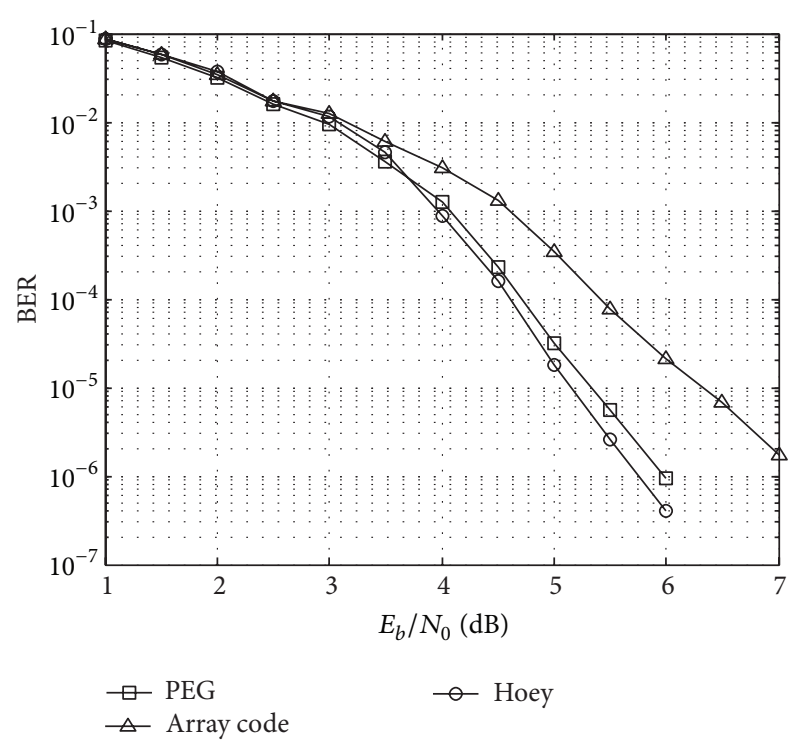

FIGURE 4: The BER curve comparison of QC-LDPC constructed by Hoey sequence, PEG algorithm codes, and array codes.

TABLE 1: The parameters of Figure 5.

\begin{tabular}{lccc}
\hline Coding type & Source node length & Code rate & Relay length \\
\hline Mackey & 5098 & 0.5 & 5098 \\
Dayan & 5568 & 0.5 & 5568 \\
Hoey & 4930 & 0.1174 & 4570 \\
Difference & 4068 & 0.5 & 4068 \\
Fibonacci & 5664 & 0.5 & 5310 \\
\hline
\end{tabular}

the channel is a Rayleigh channel, and the relevant parameters are shown in Table 1.

Figure 5 shows that the BER performance of the Mackey code which is random configuration method is inferior to the structured LDPC code. When the length of Mackey code is longer, its generating matrix and parity check matrix are more complex. Integer sequence constructed QC-LDPC codes are structural characteristics and effectively avoid the parity check matrix of the short loop. The results show that the performance of Dayan constructed QC-LDPC code is the most outstanding one.

\section{Conclusions}

In this paper, four different integer sequences were introduced and compared. The design of QC-LDPC constructed by four different sequences was used in the coded cooperative communication. Through the theoretical analysis and simulation results, it can be seen that the application of QC-LDPC codes constructed by integer sequence in the cooperative communication system is effective. Besides, the coding structure is simple and the overall performance is better than that of the other types of LDPC codes. Moreover, QC-LDPC constructed by Dayan integer sequence presents the best performance. 


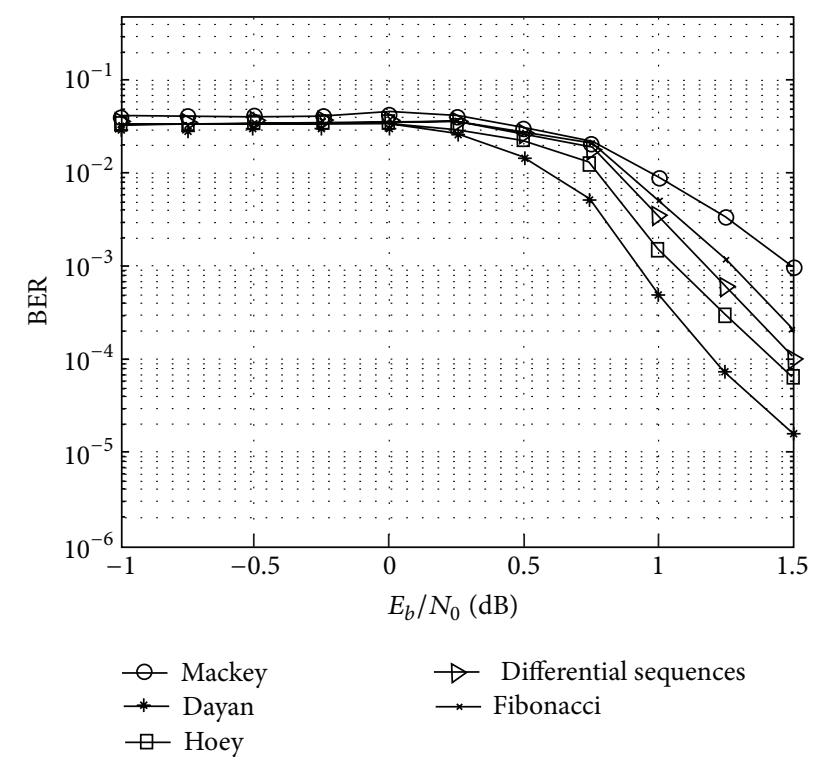

FIGURE 5: The BER performance comparison curve of QC-LDPC constructed by four different integer sequences and Mackey code in the code cooperative communication system.

\section{Conflict of Interests}

The authors declare that there is no conflict of interests regarding the publication of this paper.

\section{Acknowledgments}

This work is supported by the Science and Technology on Avionics Integration Laboratory and National Aeronautical Science Foundation of China under Contract no. 20105552.

\section{References}

[1] E. C. van der Meulen, "Three-terminal communication channels," Advances in Applied Probability, vol. 3, pp. 120-154, 1971.

[2] T. M. Cover and A. A. El Gamal, "Capacity theorems for the relay channel," IEEE Transactions on Information Theory, vol. 25, no. 5, pp. 572-584, 1979.

[3] A. Sendonaris, E. Erkip, and B. Aazhang, "User cooperation diversity. Part I: system description," IEEE Transactions on Communications, vol. 51, no. 11, pp. 1927-1938, 2003.

[4] J. N. Laneman, D. N. C. Tse, and G. W. Wornell, "Cooperative diversity in wireless networks: efficient protocols and outage behavior," IEEE Transactions on Information Theory, vol. 50, no. 12, pp. 3062-3080, 2004.

[5] T. E. Hunter and A. Nosratinia, "Cooperation diversity through coding," in Proceedings of the IEEE International Symposium on Information Theory, pp. 220-221, July 2002.

[6] P. Razaghi and W. Yu, "Bilayer low-density parity-check codes for decode-and-forward in relay channels," IEEE Transactions on Information Theory, vol. 53, no. 10, pp. 3723-3739, 2007.

[7] J. P. Canees and V. Meghdadi, "Optimized low density parity check codes designs for half duplex relay channels," IEEE Transactions on Wireless Communications, vol. 8, no. 7, pp. 3390-3395, 2009.
[8] C. X. Li, G. S. Yue, M. A. Khojastepour, X. Wang, and M. M. Madihian, "LDPC-coded cooperative relay systems: performance analysis and code design," IEEE Transactions on Communications, vol. 56, no. 3, pp. 485-496, 2008.

[9] D. Bing and Z. Jun, "Design and optimization of joint networkchannel LDPC code for wireless cooperative communications," in Proceedings of the 11th IEEE Singapore International Conference on Communication Systems (ICCS '08), pp. 1625-1629, November 2008.

[10] L. Tang, F. F. Yang, S. W. Zhang, and H. Xu, "Joint iterative decoding for LDPC-coded multi-relay cooperation with receive multi-antenna in the destination," IET Communications, vol. 7, no. 1, pp. 1-12, 2013.

[11] G. H. Zhang, R. Sun, and X. M. Wang, "Construction of girtheight QC-LDPC codes from greatest common divisor," IEEE Communications Letters, vol. 17, no. 2, pp. 369-372, 2013.

[12] X. Jiang and M. H. Lee, "Large girth quasi-cyclic LDPC codes based on the chinese remainder theorem," IEEE Communications Letters, vol. 13, no. 5, pp. 342-344, 2009.

[13] N. J. Sloane, "The on-line encyclopedia of integer sequences," Notices of the American Mathematical Society, vol. 50, no. 8, pp. 912-915, 2003.

[14] S. Zhang, F. Yang, and P. Zong, "LDPC-coded cooperative system based on joint iterative decoding," Journal of Southwest Jiaotong University, vol. 46, no. 3, pp. 469-487, 2011.

[15] M. P. C. Fossorier, "Quasi-cyclic low-density parity-check codes from circulant permutation matrices," IEEE Transactions on Information Theory, vol. 50, no. 8, pp. 1788-1793, 2004.

[16] G. Zhang, R. Sun, and X. Wang, "Construction of girtheight QC-LDPC codes from greatest common divisor," IEEE Communications Letters, vol. 17, no. 2, pp. 369-372, 2013.

[17] X. Q. Jiang and M. H. Lee, "Large girth quasi-cyclic LDPC codes based on the chinese remainder theorem," IEEE Communications Letters, vol. 13, no. 5, pp. 342-344, 2009.

[18] Y. H. Liu, X. M. Wang, R. W. Chen, and Y. He, "Generalized combining method for design of quasi-cyclic LDPC codes," IEEE Communications Letters, vol. 12, no. 5, pp. 392-394, 2008.

[19] J. Y. Kang, Q. Huang, L. Zhang, B. Zhou, and S. Lin, "Quasicyclic LDPC codes: an algebraic construction," IEEE Transactions on Communications, vol. 58, no. 5, pp. 1383-1396, 2010.

[20] W. M. Tam, F. C. M. Lau, and C. K. Tse, "A class of QCLDPC codes with low encoding complexity and good error performance," IEEE Communications Letters, vol. 14, no. 2, pp. 169-171, 2010.

[21] M. P. Fossorier, "Quasi-cyclic low-density parity-check codes from circulant permutation matrices," IEEE Transactions on Information Theory, vol. 50, no. 8, pp. 1788-1793, 2004.

[22] W. Zhan, G. X. Zhu, and L. Peng, "Design of QC-LDPC codes using Fibonacci sequence," Journal of Huazhong University of Science and Technology (Natural Science), vol. 36, no. 10, pp. 6365,2008 .

[23] L. Zhu, H. Wang, Y. Shi, and T. Xing, "Research on base matrix construction of LDPC code," Journal of Computational Information Systems, vol. 8, no. 4, pp. 1515-1521, 2012.

[24] B. Li, L. Zhang, and L. L. Cheng, "A class of $(3, \mathrm{k})$ quasicyclic LDPC codes from difference sequences with girth 8," in Proceedings of the 4th IET International Conference on Wireless, Mobile and Multimedia Networks (ICWMMN '11), pp. 108-113, November 2011.

[25] N. J. A. Sloane, "The on-line encyclopedia of integer sequences," in Towards Mechanized Mathematical Assistants, vol. 4573 of 
Lecture Notes in Computer Science, pp. 130-134, Springer, Berlin, Germany, 2007.

[26] L. Bin, Design of QC-LDPC codes based on integer sequence [M.S. thesis], Beijing Jiaotong University, Beijing, China, 2011.

[27] L. Xingcheng and C. Haohui, "Study on the construction method of quasi-cyclic LDPC codes based on progressive edgegrowth (PEG) algorithm," Journal of Circuits and Systems, vol. 14, pp. 115-119, 2009. 


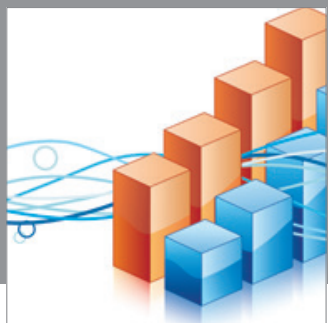

Advances in

Operations Research

mansans

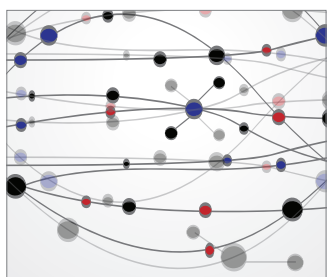

The Scientific World Journal
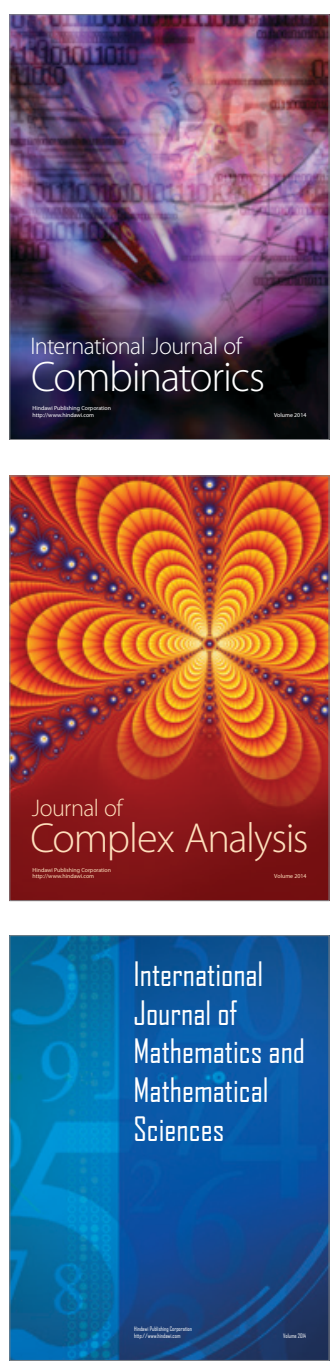
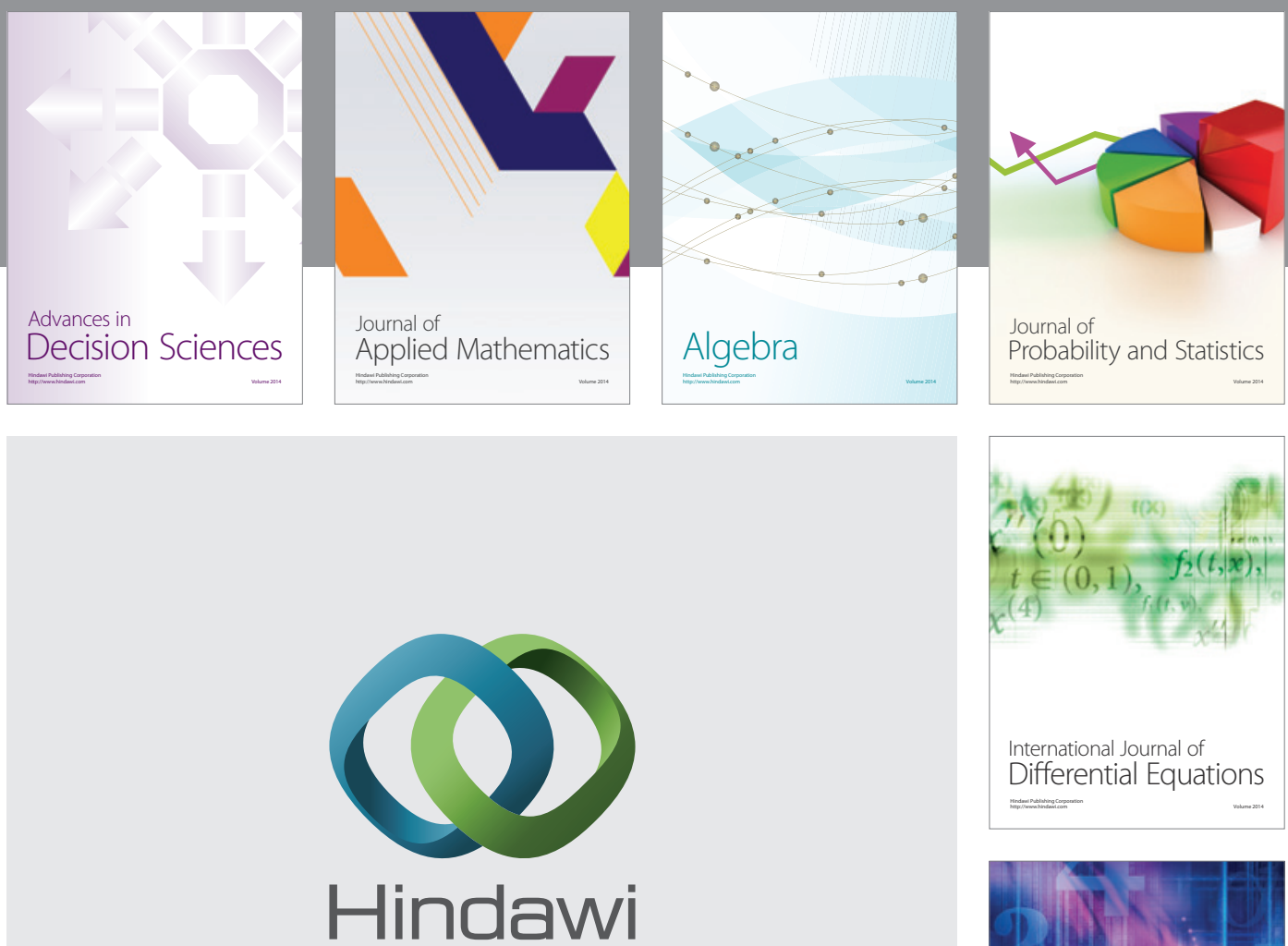

Submit your manuscripts at http://www.hindawi.com
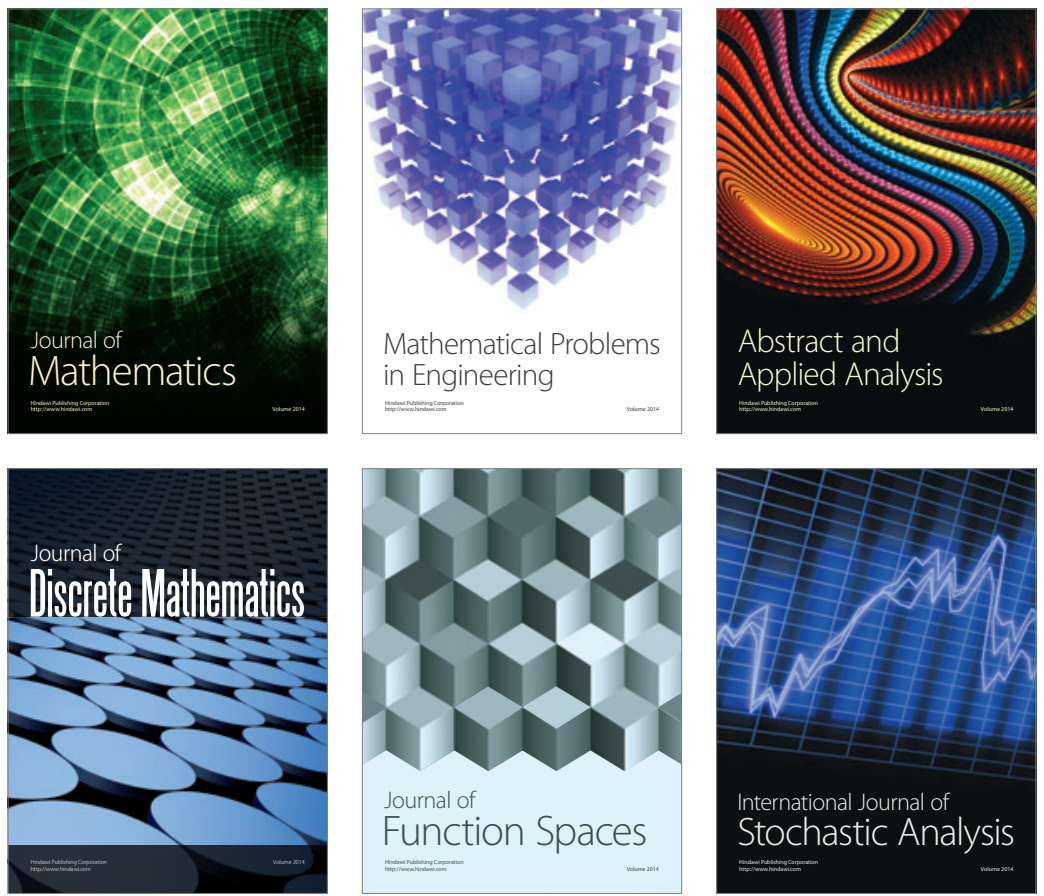

Journal of

Function Spaces

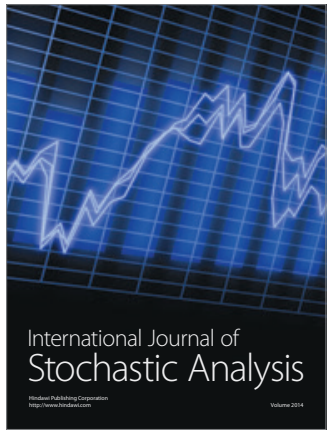

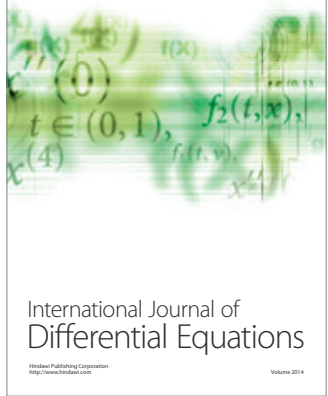
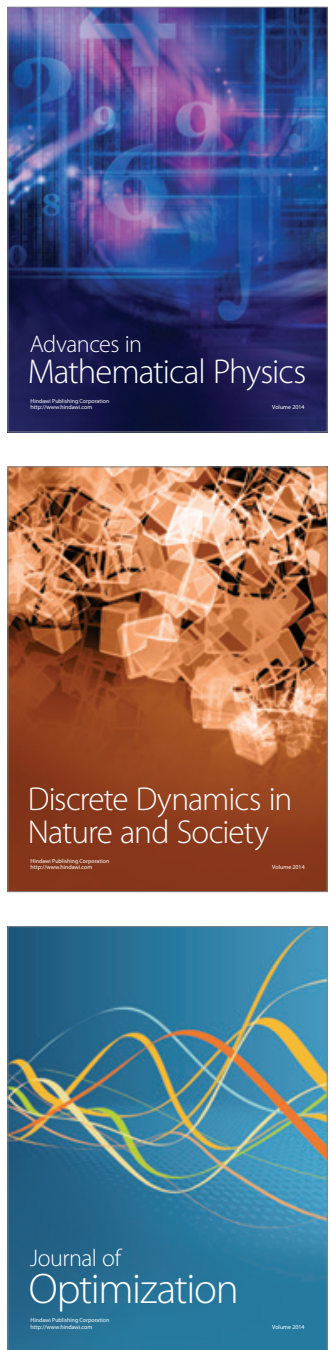